\title{
Improving the Classification of Medically Unexplained Symptoms in Primary Care
}

\author{
M. Rosendal, PhD, MD, Senior Researcher* \\ P. Fink, MD, Dr Med Sci, PhD, Senior \\ Lecturer, Research Director** \\ Erik Falkoe, MD*** \\ Henriette Schou Hansen, MD** \\ F. Olesen, MD, Dr Med Sci, Prof, Research \\ Director ${ }^{*}$ \\ * Research Unit and Institute for General \\ Practice, Aarhus University \\ ** Research Clinic for Functional Disorders, \\ Aarhus University Hospital \\ *** Research Unit for General Practice, \\ University of Southern Denmark
}

\begin{abstract}
Background: Many patients in primary care complain of physical symptoms not attributable to any known conventionally defined disease, i.e. medically unexplained symptoms (MUS).

Objectives: This paper aims to present the problems with our current classification of MUS in general practice and propose new criteria for the classification of Medically Unexplained Symptoms in a future edition of the International Classification of Primary Care (ICPC).

Methods: Discussion of European classification systems in relation to current evidence about MUS in primary care.

Results: At present, clinical care and research are hampered by the lack of a valid and reliable diagnostic classification of MUS. A particular problem in primary care is that the diagnostic category of somatoform disorders only includes persistent cases and therefore offers no opportunity for the classification of many patients with MUS in general practice. We propose new diagnostic criteria for MUS that can easily be integrated in a future edition of the ICPC. The criteria introduce mild to moderate MUS into the chapter of general and unspecified health problems if the patient has at least three MUS during an episode of care, whereas severe conditions are kept in the psychological chapter under the diagnoses applied until now.

Conclusion: A diagnosis and classification of MUS is essential for the prediction of prognosis and the choice of appropriate care for these patients in general practice. It remains to be evaluated in epidemiologic research whether the proposed classification criteria fulfil this purpose.
\end{abstract}




\section{Introduction}

It has become increasingly clear that our present diagnoses and classifications of Medically Unexplained Symptoms (MUS) do not match the problems presented by patients in primary health care ${ }^{1-3}$. Fundamental issues on classification of MUS in general practice need to be better described and become more operational in order to improve clinical care, education and research in this field.

This article will present the clinical picture in general practice and some of the problems encountered when the current European classification is used for patients with MUS in this setting. Finally, we will present new criteria for MUS that could be included in a future edition of the International Classification of Primary Care ${ }^{4}$.

\section{The picture of Medically Unexplained Symptoms in primary care}

The concept MUS may be defined as "conditions where the patient complains of physical symptoms that cause excessive worry or discomfort or lead the patient to seek treatment but for which no adequate organ pathology or patho-physiological basis can be found ${ }^{5}$. This definition has been chosen because it is purely descriptive but other names and definitions exist $^{6,7}$.

The experience of bodily sensations and symptoms is common ${ }^{8}$, and most people handle their symptoms without the involvement of the health care system. The minority of patients (about 30\%) who seek help are mainly cared for in primary care (about
$96 \%$ ). Only about $1 \%$ of people with physical symptoms are seen in specialised care ${ }^{8}$. The general practitioner (GP) is working in the borderland between normality and severe illness and therefore most of the symptoms met in primary care don't fit into the classification systems developed in specialised settings.

We may see MUS as a spectrum of disorders ranging from mild physical symptoms to serious conditions that cause suffering and disablement ${ }^{9}$. At some point in this spectrum the symptoms will have an impact that makes the affected person visit the health care system - usually a GP. At yet another point the impact is so severe that the patient is better cared for in a specialised setting. Table I presents vignettes representing this spectrum of MUS in general practice.

The prevalence depends on which part of the spectrum is under study. Kroenke and Mangelsdorff studied common physical symptoms among patients in an outpatients' clinic in the US. Seventy-four percent of these symptoms remained medically unexplained during 3 years of follow-up ${ }^{10}$. The ICD-10 criteria for somatoform disorders (criteria in Table II) have been applied in the GPs' waiting rooms in European studies. The prevalence of somatoform disorders was $16-35 \%$ and somatisation disorder $6-10 \%^{3,11}$. If the 6 month limit of symptom duration was ignored, somatoform symptoms were encountered in $60 \%$ of all patients ${ }^{3}$ matching the findings in the US study. However, we do not know whether the affected patients actually saw their GPs for their somatoform symptoms or the reason for encounter was something different. GPs have been asked to estimate the prevalence of significant MUS. On average, they registered MUS in $10-20 \%$ of patients ${ }^{2,12}$, but between GPs 


\section{Table I}

Patient stories from general practice reflecting the spectrum of Medically Unexplained Symptoms.

Case 1: Anna

Anna was a girl in her late teens and experienced quite painful stomach aches one morning. She was worried, and so was her mother, as Anna had never experienced such pains before. They decided to go and see me. I found no signs of any physical disease and reassured both of them. They were relieved when they left the consultation and I did not see Anna again for quite a while. Half a year later she came for contraceptive pills, and I asked her about her stomach. She had almost forgotten about the incident, but told me that the pains had stopped after a few days and she had been well ever since.

Diagnostic codes

ICPC-2: D02 (abdominal pain epigastric) and/or D27 (fear of digestive disease other)

ICD-10: R10.1 (pain localized to upper abdomen)

Future ICPC: as above D02 and/or D27

Case 2: Helen

Helen was 26 and she had been well until recently. Just like Anna she had stomach pain, but she was also afraid that she could have a peptic ulcer or maybe stomach cancer. The first time, she came to see me, her pains had been persistent for several weeks, and she had developed dyspeptic symptoms and started vomiting. Clinical examination revealed no organic cause of the symptoms.

About a month later she came to see me again. This time she had headaches. The headaches were accompanied by abdominal symptoms which were difficult to distinguish from her previous dyspeptic complaints.

Furthermore, it appeared that she also had severe problems at her present job. She agreed to make a symptom diary and over a few consultations we discussed the association of her symptoms with her job situation. She finally decided to quit her job and soon hereafter her symptoms disappeared.

Diagnostic codes

ICPC-2, first consultation: D02 (abdominal pain epigastric), D07 (dyspepsia/ indigestion), D10 (vomiting) and D26 (fear of cancer of digestive system)

ICPC-2, second consultation: as above D02, D07, D10 and N01 (headache), P02 (acute stress reaction), Z05 (work problem)

ICD-10, second consultation: R10.1 (pain localized to upper abdomen), R12 (heartburn), R11 (nausea and vomiting), R51 (headache), Z73 (problems related to life-management difficulty), Z56 (problems related to employment and unemployment)

Future ICPC: A24 (MUS) and as above D02, N01, Z05

Case 3: Peter

Peter came to see me every month. He was 40 years old when I first saw him. He complained about chronic backaches and headaches and had changed his listing to our practice because the previous doctor could not help him. His symptoms had been present for about 15 years and he had received all kinds of pain killers and other medication without relief - or with only very short lived effect. He had changed doctors quite a few times before. Intensive rehabilitation had been tried in his twenties but without any recovery. About 10 years ago he gave up education and work and he received a disability pension. He is now completely invalidated by his symptoms and has suffered some iatrogenic harm during the years by the provision of numerous examinations and drugs.

Diagnostic codes

ICPC-2: P75 (somatization disorder)

ICD-10: F45.0 (somatization disorder)

Future ICPC: P75 (somatoform disorder - predominantly physical symptoms) 
there was a 10-fold variation in their diagnoses $^{2}$. This variation was not explained by differences in patient populations, but was interpreted as a reflection of the GPs' different conception of where the diagnosis of MUS is placed in the spectrum of disorders.

\section{Why we need a diagnosis and classification of Medically Unexplained Symptoms}

GPs are often taught that they need to be aware of the "low prevalence epidemiology" in primary care - referring to the low prevalence of most organic diseases. Yet, at the same time GPs are also working in a high prevalence area of normal reactions and MUS and they must be aware of different implications of bodily symptoms than simply the presence or absence of disease. A diagnostic framework that includes the classification of MUS would provide construct that would help GPs conceptualize and communicate about these high prevalence phenomena. A very important aim of such a construct would be to acknowledge the problem and ensure professional and appropriate care for patients with MUS (clinical perspective). Furthermore, a diagnosis may enable quality improvement initiatives and rigorous research in the complex problems presented by MUS (research perspective). Finally, diagnoses may serve other purposes (patient perspective, social benefits etc.) which we have to bear in mind, but they are not our primary focus in this article.

\section{How Medically Unexplained Symptoms are currently classified}

\section{The classification systems}

WHO's International Classification of Disease (ICD) was developed in 1948 and is now in its $10^{\text {th }}$ version. It is used throughout the health care systems in Europe, including primary care in some countries ${ }^{13}$, and it is also used as a golden standard for primary care research. WHO has provided a brief primary care version (ICD-10-PHC) that corresponds to the ICD-10 ${ }^{14}$, but this version has not been widely implemented in European general practice. The predominant system in European primary care is the International Classification of Primary Care (ICPC) developed by the World Organisation of Family Doctors (Wonca) in 1987 and recognised as a WHO related classification for primary care in $2003^{13}$. The ICPC aims to include diagnoses of yet unclarified and complex problems. The system is biaxial and contains 17 chapters (14 biomedical, one general, one psychological and one social) and 7 components (complaints or symptom diagnoses (1-29), process codes for prophylaxis, procedures, results, administration and referrals (30-69), and specific diagnoses (70-99). Component 1 (symptoms/complaints) and component 7 (diagnosis/disease) are mapped to ICD-10 and each rubric are provided with in- and exclusion criteria in the newest edition ICPC- $2^{4}$.

Other classification systems such as the Systematized Nomenclature of Medicine, Clinical Terms (SNOMED CT) and Read Codes are also in use ${ }^{13}$, and in the United States mental disorders are classified in a separate system: the Diagnostic and Statistical Manual for Mental Disorders (DSM-4) ${ }^{15}$ or the corresponding primary care version. 
The focus of this article is the ICD- and ICPC-systems.

\section{The classification of Medically Unexplained symptoms}

Both the ICD-10, ICD-10-PHC and the ICPC-2 classify MUS either in the psychiatric chapter or as syndrome diagnoses based on predefined symptom checklists and the exclusion of organ-pathology (Table II). However, the ICD and the corresponding specific diagnoses in the ICPC have been developed in specialized settings and therefore tend to be occupied with the extreme and chronic manifestations of MUS $^{9}$. For example, symptom duration must be at least 6 months to fulfil the criteria for somatoform disorders. In a Danish study $76 \%$ of patients had shorter symptom duration according to the GPs' assessments ${ }^{16}$. Likewise, studies on health anxiety in primary care have shown that the 6 month duration criteria has been arbitrarily defined ${ }^{17}$. Consequently, the current classification leaves many primary care patients with MUS undiagnosed.

Moreover, the problem may be even more profound as it is now widely acknowledged among specialists in the field that "somatoform disorder" has failed as a diagnostic grouping ${ }^{18,19}$. In the ICPC this specific diagnosis is labelled P75 and involves the same problems as the ICD-10 diagnosis but furthermore, there is a problem with correspondence. P75 is named "somatisation disorder" in the ICPC-2 but actually includes all somatoform disorders, and the duration limit has been changed to 12 months instead of 6 months (Table II).

In conclusion, the ICD-10 systems and the specific diagnoses in the ICPC-2 cover only the minority of patients with severe MUS in primary care. Most patients with MUS and short symptom duration will be classified in the very heterogeneous group of symptom diagnoses or syndrome diagnoses.

\section{Proposal for a new classification of Medically Unexplained Symptoms in the ICPC}

A disorder is characterised by aetiology, symptom pattern, course, prognosis and outcome. With regard to MUS, problems are fundamentally heterogeneous and multi-factorial and the approach to classification will have to be pragmatic ${ }^{18}$ to ensure that the classification is acceptable and helpful to clinicians and patients. A relevant focus could be the classification's ability to predict prognosis -e.g. impairment/disability and health behaviours- and to assist the doctor in his/her choice of available treatment approaches.

In a previous article, we grouped patients with MUS according to severity (Fig. 1-2) ${ }^{9}$. Health advice refers to symptoms without significant impact on functioning or wellbeing and no indication for treatment or further investigations (example Anna, Table I). Natural (dis)stress reaction are reactions to life events and the distress does not exceed what would be expected from exposure to the stressor. Undifferentiated (dis)stress disorder or adjustment disorder includes patients who present non-specific physical, cognitive, emotional or behavioural symptoms with marked distress or significant impairment in social, occupational functioning or well-being (example Helen, Table I). Finally, the differentiated disorders are basically those classified in the ICD-10 and also 
Table II

Diagnostic criteria applicable to Medically Unexplained Symptoms in ICD-10, DSM-IV and ICPC-2.

ICD-10

Somatoform Disorders $=$ F45

Physical symptoms and persistent requests for medical investigations, in spite of negative findings and reassurance. Symptom duration $\geq 6$ months

F45.0 Somatization disorder (symptom duration $\geq 2$ years)

F45.1 Undifferentiated somatoform disorder

F45.2 Hypochondriacal disorder

F45.3 Somatoform autonomic dysfunction

F45.4 Persisting somatoform pain disorder

F45.8 Other somatoform disorders

F45.9 Somatoform disorder, unspecified

F44 Dissociative disorder
DSM-IV
Somatoform Disorders $=300$

Physical symptoms that no diagnosable general medical condition fully can account for. The symptoms cause clinically significant distress or impairment.

300.81 Somatization disorder (at least eight unexplained symptoms, duration $\geq 2$ years)

300.81 Undifferentiated somatoform disorder

300.7 Hypochondriasis

307.8 Pain disorder

300.7 Body dysmorphic disorder

300.81 Somatoform disorder NOS

300.11 Conversion disorder
Related diagnoses

F22.8 Persist. delusional disorders

F48.0 Neurasthenia

F68.1 Factitious disorder

Syndrome diagnoses as for example

K58 Irritable bowel syndrome
Related diagnoses

300.1 Factitious disorder

ICPC-2

Somatization disorder $=\mathrm{P} 75$

Persisting and multiple physical symptoms and demands of examinations despite negative results and reassurance from the doctor.

Symptom duration $>12$ months
Related diagnoses
P02: Acute stress reaction
P29: Psychological symptom/
complaint, other
P78: Neurasthenia
P99: Psychological disorders,
other
Specific syndrome diagnoses as
for example
D93: Irritable bowel syndrome
Symptom diagnoses (1-29)
from all organ chapters A to Y include somatisation disorder in the ICPC (example Peter, Table I).

The ICPC already lays emphasis on codes for symptom diagnoses and these codes may be used for unexplained symptoms (A-Y 1-29). However, the symptom diagnoses also include symptoms under investigation, banalities or minor ailments where the GP find no indication for further intervention, along with mild MUS and persistent symptoms that remain unexplained (Figure 1). What we need is a more specific category for the unexplained symptoms that are neither self-limited nor fulfilling the criteria for persistent disorders. The challenge is how we distinguish self-limiting symptoms from clinical significant MUS.

Previous studies have demonstrated that the number of symptoms may be an indicator of severity and relates to physical functioning and health care utilization ${ }^{10,20-23}$. But the associations show no clear cut off for subgroups of severity and the number of symptoms chosen for classification will therefore be arbitrary. Previous studies have also specified particular symptoms related to somatoform disorders ${ }^{21,22,24}$. In line with this, a new empirical study about classification of bodily distress suggests that a minimum of 3 "non-specific symptoms" from a specified list form the diagnostic criteria ${ }^{23}$. 


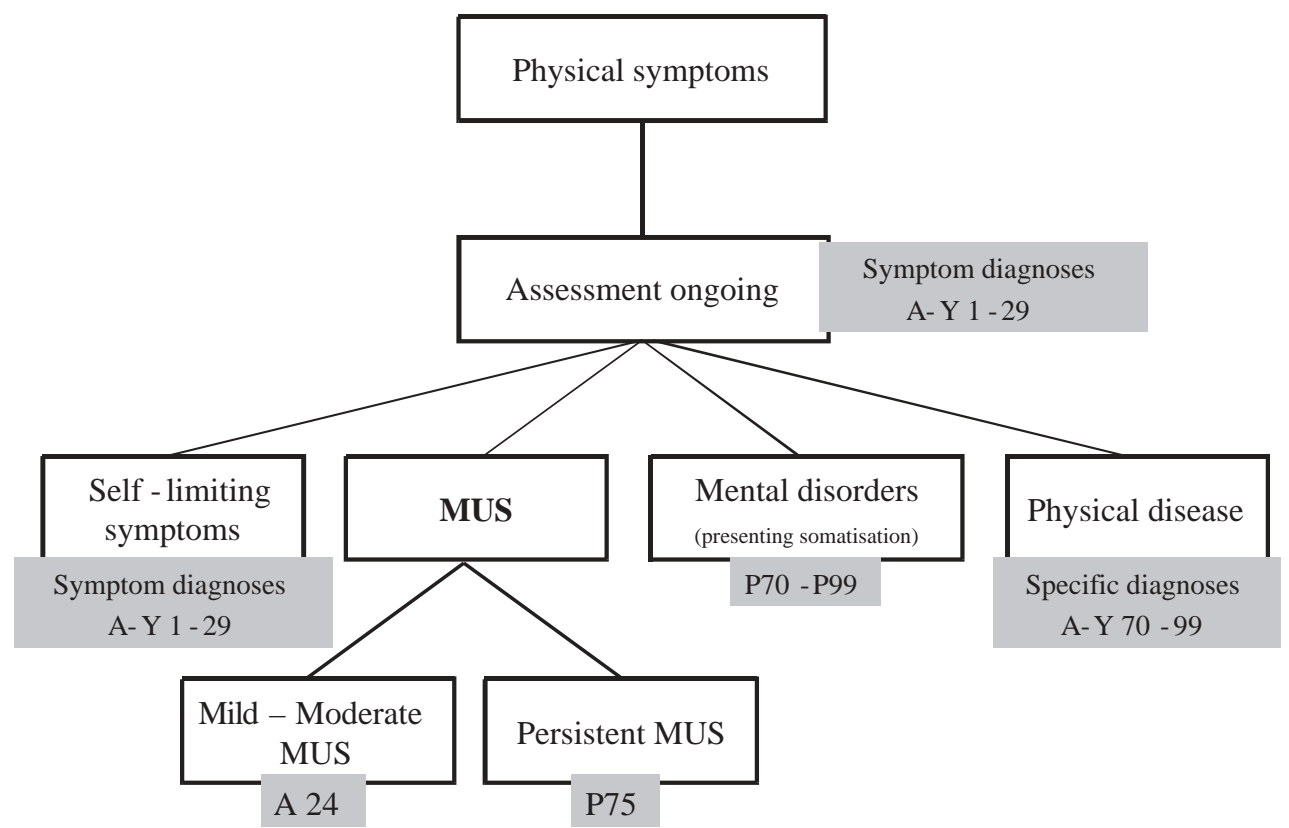

Figure 1. A categorization of physical symptoms and the corresponding diagnostic codes, as they could be defined in a future edition of the ICPC.

\section{Clinical diagnosis $\quad$ ICPC codes}

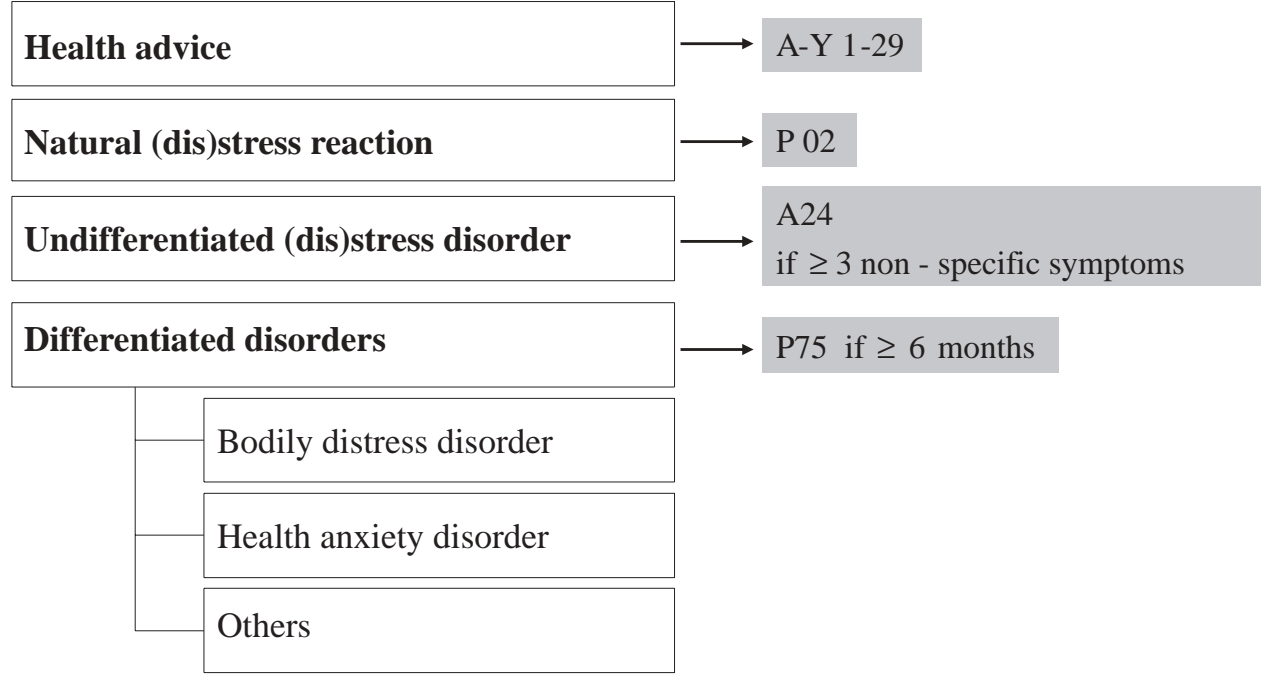

Figure 2. A classification of Medically Unexplained Symptoms in general practice (Fink et al. ${ }^{9}$ ). The proposed diagnostic codes from a future edition of the ICPC have been added. 
This cut-point was chosen with a clinical perspective of specificity in mind. On this basis, we suggest an arbitrary cut-point of 3 symptoms for the diagnosis "MUS". Nonspecific symptoms are found among the symptom diagnoses in the ICPC, but some symptom diagnoses may actually be specific (for example D13 jaundice). A list of nonspecific symptom diagnoses would increase the reliability of the MUS diagnosis but implementation will depend on easy access via computer systems (Table III).

Besides symptom count, duration serves as a relevant distinction between mild and severe conditions. We have maintained the 6 months limit to separate mild from moderate conditions.

The aetiology of MUS is multi-factorial and in many cases unknown and complex. Although, psychological and social factors play an important role in some cases; they are, contrary to many doctors' conviction, not a precondition to classify symptoms as medically unexplained. Thus, the mild con- ditions in general practice do not naturally fall into the psychiatric chapter in the classification. Instead, we have maintained a descriptive definition and propose to place it in chapter A, general and unspecified health problems, in a future edition of the ICPC.

In conclusion, we suggest a description and classification of MUS relating to severity as described in detail in Table IV. We may also have to reconsider the name, and a suggestion is "Multiple Idiopathic Physical Symptoms". Furthermore, our proposal includes a change in the name and duration limit for somatisation disorder in the ICPC-2 in order to make this diagnosis correspond to the ICD-10. Finally, we suggest that somatoform disorders are split into two main categories: predominantly physical complaints and predominantly health anxiety (previous hypochondriacal disorder). The latter would include the most severe cases of MUS on the anxiety dimension and thus form the final point in the spectrum of symptom diagnoses describing fear of...

Table III

Chapters of the ICPC-2 and a list of the symptom diagnoses which may reflect unspecific symptoms.

A: General and Unspecified

A01 pain general/multiple sites, A02 chills, A04 weakness/tiredness general, A05 feeling ill, A09 sweating problem, A11 chest pain NOS, A25 fear of death/dying, A26 fear of cancer NOS, A27 fear of other disease NOS

B: Blood, Blood Forming Organs and Immune Mechanism

B25 fear of aids/HIV, B26 fear cancer blood /lymph, B27 fear blood/lymph disease other

D: Digestive

D01 abdominal pain, D02 abdominal pain epigastric, D03 heartburn, D04 rectal/anal pain, D05 perianal itching, D07 dyspepsia/indigestion, D08 flatulence/gas/belching, D09 nausea, D10 vomiting (other than during pregnancy), D11 diarrhoea, D12 constipation, D18 change faeces/bowel movements, D20 mouth/tongue/lip symptom/complaint, D21 swallowing problem, D25 abdominal distension, D26 fear of cancer of digestive system, D27 fear of digestive disease other

F: Eye

F13 eye sensation abnormal, F27 fear of eye disease

H: Ear

$\mathrm{H} 27$ fear of ear disease 
Table III. (Continue)

K: Cardiovascular

K02 pressure/tightness of heart, K04 palpitations/awareness of heart, K24 fear of heart disease, K25 fear of hypertension, K27 fear cardiovascular disease other

L: Musculoskeletal

L01 neck symptom/complaint, L02 back symptom/complaint, L03 low back symptom/complaint, L04 chest symptom/complaint, L05 flank/axilla symptom/complaint, L07 jaw symptom/complaint, L09 arm symptom/complaint, L12 hand/finger symptom/complaint, L14 leg/thigh symptom/complaint, L17 foot/toe symptom/complaint, L18 muscle pain, L19 muscle symptom/complaint NOS, L20 joint symptom/complaint NOS, L26 fear of cancer musculoskeletal, L27 fear musculoskeletal disease other

$\mathrm{N}$ : Neurological

N01 headache, N02 pain face, N04 restless legs, N05 tingling fingers/feet/toes, N06 sensation disturbance other, N08 abnormal involuntary movements, N16 disturbance of smell/taste, N17 vertigo/dizziness, N19 speech disorder, N26 fear cancer neurological system, N27 fear of neurological disease other

P: Psychological

P01 feeling anxious/nervous/tense, P02 acute stress reaction, P06 sleep disturbance, P20 memory disturbance

R: Respiratory

R04 breathing problem, other, R21 throat symptom/complaint, R26 fear of cancer respiratory system, R27 fear of respiratory disease, other, R98 hyperventilation

S: Skin

S01 pain/tenderness of skin, S02 pruritus, S08 skin colour change, S26 fear of cancer of skin, S27 fear of skin disease other

T: Endocrine/Metabolic and Nutritional

T03 loss of appetite, T26 fear of cancer of endocrine system, T27 fear endocrine/metabolic dis. Other

U: Urological

U07 urine symptom/complaint other, U13 bladder symptom/complaint other, U26 fear of cancer of urinary system, U27 fear of urinary disease other

W: Pregnancy, Childbearing, Family Planning

W27 ear complications of pregnancy

$\mathrm{X}$ : Female Genital

X01 genital pain female, X15 vaginal symptom/complaint other, X16 vulval symptom/complaint, X17 pelvis symptom/complaint, X18 breast pain, X21 breast symptom/complaint other, X23 fear sexually transmitted disease, X24 fear of sexual dysfunction, X25 fear of genital cancer, X26 fear of breast cancer, X27 fear genital/breast disease other

Y: Male Genital

Y01 pain in penis, Y02 pain in testis/scrotum, Y04 penis symptom/complaint other, Y05 scrotum complaint other, Y06 prostate symptom/complaint, Y16 breast symptom/complaint, Y24 fear of sexual dysfunction, Y25 fear sexually transmitted disease, Y26 fear of genital cancer, Y27 fear genital disease other

Z: Social Problems

Note: Symptoms in Italic have been verified to be associated with MUS in a recent empirical study (Fink et al. ${ }^{23}$ ), although the ICPC-rubrics may be more comprehensive and thus include a broader spectrum of symptoms than those in the study. 


\section{Discussion}

The presented classification (Table IV) includes mild-moderate conditions in addition to the severe conditions from our present classification systems. It is based on a descriptive approach and classifies milder conditions as general and unspecified health problems rather than psychological problems. This placement emphasizes the uncertain nature of mild MUS and makes the diagnosis more useful to GPs and less stigmatizing to patients. The proposed classification has not been empirically validated and it has yet to be evaluated whether it relates to disability and is able to predict prognosis and assign relevant treatment approaches (for example specific treatment for chronic disorders, reattribution models for moderate conditions and reassurance/ normalisation for mild conditions). An evaluation should explore the conceptualization of the classification and the arbitrarily set cut points of 3 symptoms, 6 months and the symptom list.

The proposed classification contains positive criteria and may not include some patients with significant idiopathic symptoms. For example patients with single disabling symptoms will not be diagnosed until

Table IV

Suggestion for a new classification of self-limiting complaints and Medically Unexplained Symptoms in the International Classification for Primary Care (ICPC).

Symptom diagnoses: A-Y 1-29 Single symptom diagnoses with expected spontaneous remission. Assessment (including watchful waiting) may be ongoing, but MUS is not suspected

Medically Unexplained Symptoms: Physical symptoms for which NO adequate organ pathology or pathoRubric in chapter A (general and uns- physiological basis can be found. pecified)

Component 1 (symptoms and complaints)

For instance A 24

The diagnosis does not imply a psychosocial aetiology but psychological and/or social strain may be contributory

At least three non-specific symptoms* at the same time or during an episode of care**

Simultaneous coding of symptom Duration does not exceed six months

diagnoses from the relevant organ Exclusion criteria: The symptoms are not better accounted for by P75 chapters

Alternative name: somatoform disorder, P78 neurasthenia, P82 post-traumatic stress disorder or another specific disorder

Multiple Idiopathic Physical Symp- Consider presenting somatisation in psychiatric disorders such as P74 toms anxiety and P76 depression

Somatoform disorder: P75

Corresponds to somatoform disorder in the ICD-10

Physical symptoms in spite of negative findings and reassurance

Duration more than six months

Somatoform disorders consist of two main categories:

A. Predominant complaints are physical symptoms (somatization disorder, undifferentiated somatoform disorder, somatoform autonomic dysfunction, persisting somatoform pain disorder and others)

B. Predominant complaint is anxiety (hypochondriacal disorder/health anxiety)

\footnotetext{
*) A non-specific symptom is not in itself specific to an identifiable disease. This category also includes symptom diagnoses "fear of...", when these diagnoses are applied to physical complaints. A list of non-specific symptom diagnoses is shown in Table III.

**) Episode of care: The period from the first presentation by the patient of a health problem or illness to the health care provider until the completion of the last encounter for the same health problem. Its name (diagnosis) may change over time.
} 
they fulfil criteria for somatoform disorders after 6 months. We need to know how large this group is and whether these patients need to be diagnosed with MUS at an earlier stage.

We have chosen a one-dimensional approach to classification despite the complex nature of MUS. Multi-axial systems would give a more comprehensive understanding of bodily symptoms as they could focus on the three aspects of the bio-psycho-social model used in general practice ${ }^{25}$. However, the suggestion to apply a multi-axial classification is not appealing in primary care. GPs' consultations are very time-limited, so naturally, they wish to minimize the time spent on administrative procedures such as diagnostic coding.

Finally, the proposed criteria must be made operational by the integration into the GPs' electronic patient records. The computer systems may be programmed to react when at least 3 non-specific symptom diagnoses from the list are active (in episodes of care). This would prompt the GP to consider the diagnosis MUS and either accept or reject it. If electronic systems are not available we will expect a more pragmatic application of the criteria in daily practice.

\section{Conclusion and perspective}

Diagnosis is derived from the Greek words dia (between) and gignoskein (to know/distinguish) meaning "to know between". In relation to MUS we may have to reconsider our paradigm for diagnosis and differentiate between different treatment approaches rather than focus on organ pathology or symptom patterns. In order to make this differentiation, we may use prognostic factors such as symptom duration and number of symptoms for the classification of subgroups.
The presented proposal for a new classification is differentiated, matches primary health care patient populations, builds on an existing classification system and is simple to understand and use. Furthermore, it provides a more balanced weight to medically unexplained symptoms and enables communication and research in primary care. However, to make progress in the care for patients with MUS we need rigorous evaluation of new classifications in empirical studies.

\section{Reference}

1. Dew K, Dowell A, McLeod D, Collings S, Bushnell J. "This glorious twilight zone of uncertainty": mental health consultations in general practice in New Zealand. Soc Sci Med 2005; 61: 1189-1200.

2. Rosendal M, Bro F, Fink P, Christensen KS, Olesen, F. Diagnosis of somatisation: effect of an educational intervention in a cluster randomised controlled trial. Br. J Gen Pract 2003; 53: 917-922.

3. Toft T, Fink P, Oernboel E, Christensen KS, Frostholm L, Olesen F. Mental disorders in primary care: prevalence and co-morbidity among disorders. Results from the Functional Illness in Primary care (FIP) study. Psychol Med 2005; 35: 1175-1184.

4. WONCA. International Classification of Primary Care. ICPC-2-R. New York: Oxford University Press; 2005.

5. Fink P, Rosendal M, Toft T. Assessment and treatment of functional disorders in general practice: the extended reattribution and management model - an advanced educational program for nonpsychiatric doctors. Psychosomatics 2002; 43: 93-131.

6. Rosendal M, Fink P, Bro F, Olesen F. Somatization, heartsink patients, or functional somatic symptoms? Towards a clinical useful classification in primary health care. Scand J Prim Health Care 2005; 23: 3-10.

7. Burton C. Beyond somatisation: a review of the understanding and treatment of medically unexplained physical symptoms (MUPS). Br J Gen Pract 2003; 53: 231-241.

8. Green LA, Fryer GE Jr, Yawn BP, Lanier D, Dovey SM. The ecology of medical care revisited. N Engl J Med 2001; 344: 2021-2025. 
9. Fink P, Rosendal M, Olesen F. The classification of somatisation and functional somatic symptoms in primary care. Aust N Z J Psychiatry 2005; 39: 772-781.

10. Kroenke K, Mangelsdorff AD. Common symptoms in ambulatory care: incidence, evaluation, therapy, and outcome. Am J Med 1989; 86: 262-266.

11. de Waal MW, Arnold IA, Eekhof J A, van Hemert AM. Somatoform disorders in general practice: prevalence, functional impairment and comorbidity with anxiety and depressive disorders. Br J Psychiatry 2004 184: 470476.

12. Peveler R, Kilkenny L, Kinmonth AL. Medically unexplained physical symptoms in primary care: a comparison of self-report screening questionnaires and clinical opinion. J Psychosom Res 1997; 42: 245-252.

13. de Lusignan S, Minmagh C, Kennedy J, Zeimet M, Bommezijn H, Bryant J. A survey to identify the clinical coding and classification systems currently in use across Europe. Medinfo 2001; 10: 86-89.

14. Ustun TB, Goldberg D, Cooper J, Simon GE, Sartorius $\mathrm{N}$. New classification for mental disorders with management guidelines for use in primary care: ICD-10 PHC chapter five. Br J Gen Pract 1995; 45: 211-215.

15. Francis A, Pincus HA, First MB. DSM-IV: Diagnostic and statistical manual of mental disorders. Washington DC: American Psychiatric Association; 1994.

16. Rosendal M., Olesen F, Fink P, Toft T, Sokolowski I, Bro F. A randomized controlled trial of brief training in assessment and treatment of somatisation in primary care: effects on patient outcome. Gen Hosp Psychiatry 2007; (accepted).

17. Fink P, Ornbol E, Toft T, Sparle KC, Frostholm L, Olesen F. A new, empirically established hypochondriasis diagnosis. Am J Psychiatry 2004; 161: 1680-1691.
18. Engel CC. Explanatory and pragmatic perspectives regarding idiopathic physical symptoms and related syndromes. CNS Spectr 2006; 11: 225-232.

19. Mayou R, Kirmayer LJ, Simon G, Kroenke K, Sharpe M. Somatoform disorders: time for a new approach in DSM-V. Am. J Psychiatry 2005; 162: 847-855.

20. Jackson J, Fiddler M, Kapur N, Wells A, Tomenson B, Creed F. Number of bodily symptoms predicts outcome more accurately than health anxiety in patients attending neurology, cardiology, and gastroenterology clinics. J Psychosom Res 2006; 60: 357-363.

21. Kroenke K, Spitzer RL, Williams JBW. The PHQ15: Validity of a new measure for evaluating the severity of somatic symptoms. Psychosom Med 2002; 64: 258-266.

22. Escobar JI, Waitzkin H, Silver RC, Gara M, Holman A. Abridged somatization: a study in primary care. Psychosom Med 1998; 60: 466-472.

23. Fink P, Toft T, Hansen MS, Oernboel E, Olesen F. Symptoms and syndromes of bodily distress. An explorative study among 978 internal medical, neurological and primary care patients. Psychosom Med 2007; (in press).

24. Derogatis LR, Lipman RS, Covi L. SCL-90: an outpatient psychiatric rating scale - preliminary report. Psychopharmacol Bull 1973; 9: 13-28.

25. Sharpe M, Mayou R, Walker J. Bodily symptoms: New approaches to classification. J Psychosom Res 2006; 60: 353-356.

Address for correspondence:

Marianne Rosendal

Research Unit for General Practice

Vennelyst Boulevard 6, DK-8000 Aarhus, Denmark

m.rosendal@dadlnet.dk

Ph: +452041 1619/+4589426010

Fax: +4586124788 